\title{
On a Certain Operator Related to Blackwell's Markov Chain
}

\author{
Ernest Nieznaj ${ }^{1}$ (D)
}

Received: 2 January 2020 / Revised: 14 May 2021 / Accepted: 25 May 2021 / Published online: 2 June 2021 (c) The Author(s) 2021

\begin{abstract}
We present an example of a densely defined, linear operator on the $l^{1}$ space with the property that each basis vector of the standard Schauder basis of $l^{1}$ does not belong to its domain. Our example is based on the construction of a Markov chain with all states instantaneous given by D. Blackwell in 1958. In addition, it turns out that the closure of this operator is the generator of a strongly continuous semigroup of Markov operators associated with Blackwell's chain.
\end{abstract}

Keywords Blackwell's example · Markov semigroup · unbounded operator

Mathematics Subject Classification (2020) 47D07 · 60J27 · 60J35

\section{Introduction}

W. Feller and H.P. McKean showed in 1956, see [3], that there exists a Markov chain with all states instantaneous. They considered a diffusion $x_{t}$ with the state space $K=[0,1]$ and with some specified transition function, and proved that this diffusion is in fact equivalent to a Markov chain $\hat{x}_{t}$ with the state space $\mathbb{N}$, where $\mathbb{N}$ is the set of natural numbers. In addition, the $Q$ matrix of the chain has $-\infty$ down the diagonal and zeroes elsewhere. Thus, the Feller-McKean chain is a continuous process which spends almost all of its time on $\mathbb{N}$ even though it leaves each state instantly.

In 1958, D. Blackwell, see [1], gave a more tractable example of a Markov chain with all states instantaneous. In this situation, the connection between intensity matrices of Markov chains and semigroups of Markov operators in $l^{1}$ corresponding to them, is of special interest. To be precise, let $X(t), t \geq 0$ be a Markov chain with values in $\mathbb{N}$ and $\{P(t), t \geq 0\}$ be its corresponding Markov semigroup in $l^{1}$ with generator $A$. Lemma 2 in [5] says that if a given state $i \in \mathbb{N}$ is instantaneous then $e_{i} \notin \mathcal{D}(A)$, where $e_{i}$ is an element of the standard Schauder basis in $l^{1}$ and $\mathcal{D}(A)$ is the domain of $A$, see Sect. 2

\footnotetext{
Ernest Nieznaj

e.nieznaj@pollub.pl

1 Lublin University of Technology, Nadbystrzycka 38A, 20-618 Lublin, Poland
} 
for definitions. Since all states of Blackwell's chain are instantaneous, $e_{i} \notin \mathcal{D}\left(A_{B}\right)$ for every $i \in \mathbb{N}$, where $A_{B}$ is the generator of the corresponding Blackwell semigroup. Because $A_{B}$ is not explicitly given in the literature (as far as I am aware), I became interested in finding a formula for this generator. However, due to difficulties with a precise description of the domains of such generators, I slightly modified my problem. It can be stated as follows: find a formula for a linear operator $A: \mathcal{D}(A) \rightarrow l^{1}$ that has the following properties

(i) $\mathcal{D}(A)$ is dense in $l^{1}$, (ii) $e_{i} \notin \mathcal{D}(A)$ for every $i \in \mathbb{N}$,

and such that explicit calculations are possible. So now we do not require $A$ to be a generator.

Theorem 1 answers our question and an example of an operator with such properties is given by (12)-(13). Moreover, Theorem 1 implies that the closure of $A$ equals $A_{B}$, i.e. $\bar{A}=A_{B}$, see Sect. 4 for details.

In the next section, we introduce basic notions and present the construction of Blackwell's semigroup. This construction is needed for us to define the operator $A$ in Sect. 3.

\section{Preliminaries}

\subsection{The Blackwell Semigroup}

We begin with some definitions. Let $l^{1}$ be the Banach space of all absolutely summable sequences $x=\left(\xi_{i}\right)_{i \in \mathbb{N}}$, i.e. sequences such that $\sum_{i \in \mathbb{N}}\left|\xi_{i}\right|<\infty$, with the norm $\|x\|=\sum_{i \in \mathbb{N}} \xi_{i}$. In this article, $\mathbb{N}=\{1,2,3, \ldots\}$. By $\left\{e_{i}\right\}_{i \in \mathbb{N}}$, we denote the standard Schauder basis in $l^{1}$, i.e. $e_{i}=(0, \ldots, 0,1,0, \ldots)$ with 1 in the $i$ th coordinate. Any $x \in l^{1}$ can be then written as $x=\sum_{i \in \mathbb{N}} \xi_{i} e_{i}$.

Throughout we assume that $\alpha_{n}, \beta_{n}, n \geq 1$ are positive numbers satisfying

$$
\sum_{n=1}^{\infty} \frac{\beta_{n}}{\alpha_{n}+\beta_{n}}<\infty, \quad \sum_{n=1}^{\infty} \beta_{n}=\infty
$$

Incidentally, this assumption implies that $\lim _{n \rightarrow \infty} \frac{\beta_{n}}{\alpha_{n}}=0$ and $\sum_{n=1}^{\infty} \frac{\beta_{n}}{\alpha_{n}}<\infty$.

Now we follow the construction of the Blackwell semigroup as described in [2]. Firstly, let $\mathbb{I}$ be the set of functions $\widetilde{i}: \mathbb{N} \rightarrow\{0,1\}$ admitting value 1 finitely many times. Elements of $\mathbb{I}$ may be considered as sequences with a finite number of nonzero terms. Since $\mathbb{I}$ is countable, the definition of $l^{1}(\mathbb{I})$ is analogous to that of $l^{1}(\mathbb{N})$. By $\left\{e_{i}^{\sim}\right\}_{\tilde{i} \in \mathbb{I}}$ we denote the natural basis in $l^{1}(\mathbb{I})$. For $n \geq 1$, let $F_{n}$ be the map $F_{n}: \mathbb{I} \rightarrow \mathbb{I}$ changing the $n$th coordinate of an $\tilde{i}$ from 0 to 1 and vice versa. The map $G_{n}: \mathbb{I} \rightarrow\{0,1\}$ assigns to an $\tilde{i}$ its $n$th coordinate. 
For $n \geq 1$, let $B_{n}$ be the bounded linear operator on $l^{1}(\mathbb{I})$ determined by its values on $e_{i}, \widetilde{i} \in \mathbb{I}$, by

$$
B_{n} e_{i}= \begin{cases}-\beta_{n} e_{i}+\beta_{n} e_{F_{n}(\tilde{i})}, & \text { if } G_{n}(\widetilde{i})=0 \\ -\alpha_{n} e_{i}^{\widetilde{1}}+\alpha_{n} e_{F_{n}(\widetilde{i})}, & \text { if } G_{n}(\widetilde{i})=1\end{cases}
$$

Then for any $x=\sum_{\widetilde{i} \in \mathbb{I}} \delta_{\tilde{i}} e_{i}^{\sim}$ from $l^{1}(\mathbb{I})$ we have $B_{n} x=\sum_{\widetilde{i} \in \mathbb{I}} \delta_{\tilde{i}} B_{n} e_{\tilde{i}}$.

As explained in [2], $B_{n}$ is the generator of a Markov chain on $\mathbb{I}$ in which the $n$th coordinate of an $\widetilde{i} \in \mathbb{I}$ jumps between 0 and 1 . In terms of semigroups, $B_{n}$ generates a Markov semigroup $\left\{e^{t B_{n}}, t \geq 0\right\}$ which values on $\left\{e_{i}\right\}_{i \in \mathbb{I}}$ are given by

$$
e^{t B_{n}} e_{i}= \begin{cases}p_{n}(t) e_{\tilde{i}}+\left(1-p_{n}(t)\right) e_{F_{n}(\widetilde{i})}, & \text { if } G_{n}(\widetilde{i})=0 \\ q_{n}(t) e_{i}+\left(1-q_{n}(t)\right) e_{F_{n}(\widetilde{i})}, & \text { if } G_{n}(\widetilde{i})=1\end{cases}
$$

where

$$
\begin{aligned}
& p_{n}(t)=\frac{\alpha_{n}}{\alpha_{n}+\beta_{n}}+\frac{\beta_{n}}{\alpha_{n}+\beta_{n}} e^{-\left(\alpha_{n}+\beta_{n}\right) t}, \\
& q_{n}(t)=\frac{\beta_{n}}{\alpha_{n}+\beta_{n}}+\frac{\alpha_{n}}{\alpha_{n}+\beta_{n}} e^{-\left(\alpha_{n}+\beta_{n}\right) t} .
\end{aligned}
$$

Since $B_{n}$ 's commute and are bounded, $T_{n}(t)=\prod_{k=1}^{n} e^{t B_{k}}$ defines a strongly continuous semigroup $\left\{T_{n}(t), t \geq 0\right\}$, with generator $A_{n}=\sum_{k=1}^{n} B_{k}$. The semigroup $\left\{T_{n}(t), t \geq 0\right\}$ describes $n$ combined independent Markov chains, each changing one of the first $n$ coordinates of $\tilde{i} \in \mathbb{I}$.

Theorem 4.2 of [2] says that semigroups $\left\{T_{n}(t), t \geq 0\right\}$ converge, when $n \rightarrow \infty$, to a strongly continuous semigroup $\{T(t), t \geq 0\}$ composed of Markov operators. In such a case, we say that the infinite product $\prod_{k=1}^{\infty} e^{t B_{k}}$ exists and denote

$$
\prod_{k=1}^{\infty} e^{t B_{k}}:=T(t)
$$

This limit semigroup is just the Blackwell semigroup defined on $l^{1}(\mathbb{I})$. It is also denoted by $e^{t A_{B}}$, where $A_{B}$ is the generator of $\{T(t), t \geq 0\}$. Although a formula for $T(t)$ is given in Lemma 4.1 of [2] it is not useful here, since it is expressed as an infinite sum of terms which are difficult to handle.

The fact that semigroups $T_{n}(t)$ converge strongly is also a conclusion from Theorem 1, see Remark 1 in Sect. 4.

\subsection{The Binary Bijection}

Here we specify a bijection between $\mathbb{N}$ and $\mathbb{I}$. By doing so, we are able to write explicit formulae for isomorphic images of $B_{n}$ 's and $A_{n}$ 's. 
Let $f(1)=\widetilde{1}:=(0,0,0, \ldots) \in \mathbb{I}$ and for $i \geq 2$ define

$$
f(i)=\widetilde{i}:=\left(j_{1}, \ldots, j_{m}, 0,0, \ldots\right)
$$

where $j_{k} \in\{0,1\}, k=1,2, \ldots, m$, are such that $\sum_{k=1}^{m} j_{k} 2^{k-1}=i-1$. This is simply to say that we assign the binary representation of $i-1$ to a number $i \geq 2$. For instance, we have

$$
f(2)=\widetilde{2}=(1,0,0,0, \ldots), \quad f(8)=\widetilde{8}=(1,1,1,0,0, \ldots), \quad \text { etc. }
$$

It is obvious that $\tilde{i} \in \mathbb{I}$ and that each element of $\mathbb{I}$ is a binary representation of a natural number. In consequence, we have a one-to-one correspondence between $l^{1}(\mathbb{N})$ and $l^{1}(\mathbb{I})$. This correspondence, written between bases $\left\{e_{i}\right\}_{i \in \mathbb{N}}$ and $\left\{e_{i}\right\}_{\widetilde{i}}, \mathbb{I}$, can be defined as follows

$$
f\left(e_{i}\right):=e_{f(i)}, \quad i \in \mathbb{N},
$$

where $f(i)$ is given by (5). Then we extend $f$ from $\left\{e_{i}\right\}_{i \in \mathbb{N}}$ to all elements of $l^{1}(\mathbb{N})$ by linearity, i.e. $f(x)=\sum_{i \in \mathbb{N}} \xi_{i} f\left(e_{i}\right)$ if $x=\sum_{i \in \mathbb{N}} \xi_{i} e_{i}$.

Using (3), we define operators $\mathcal{B}_{n}, n \geq 1$, on $l^{1}(\mathbb{N})$ as follows. Firstly, define

$$
\mathcal{B}_{n} e_{i}:=f^{-1}\left(B_{n} f\left(e_{i}\right)\right), \quad \text { for } i \in \mathbb{N}
$$

and $\mathcal{B}_{n} x=\sum_{i \in \mathbb{N}} \xi_{i} \mathcal{B}_{n} e_{i}$ for any $x \in l^{1}(\mathbb{N})$.

Lemma 1 If $\left(\eta_{i}\right)_{i \in \mathbb{N}}=\mathcal{B}_{n}\left(\xi_{i}\right)_{i \in \mathbb{N}}$, then

$$
\eta_{i}=\left\{\begin{array}{l}
-\beta_{n} \xi_{i}+\alpha_{n} \xi_{i+2^{n-1}}, \\
\quad \text { if } i \bmod 2^{n} \in\left\{1,2, \ldots, 2^{n-1}\right\}, \\
-\alpha_{n} \xi_{i}+\beta_{n} \xi_{i-2^{n-1}} \\
\quad \text { if } i \bmod 2^{n} \in\left\{0,2^{n-1}+1, \ldots, 2^{n}-1\right\} .
\end{array}\right.
$$

Proof Fix $n \geq 1$. To find an explicit formula for $\mathcal{B}_{n}$ notice that for $\widetilde{i}=f(i)$, where $i \in \mathbb{N}$, we have

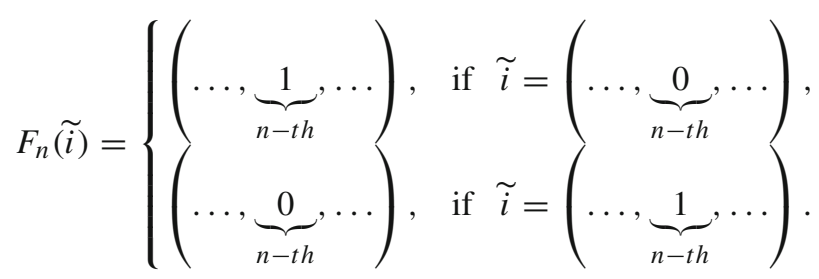


From this, we immediately obtain

$$
f^{-1}\left(F_{n}(f(i))= \begin{cases}i+2^{n-1}, & \text { if } f(i)=(\ldots, \underbrace{0}_{n-t h}, \ldots), \\ i-2^{n-1}, & \text { if } f(i)=(\ldots, \underbrace{1}_{n-t h}, \ldots) .\end{cases}\right.
$$

Next, we find $G_{n}(f(i))$. The binary arithmetic with some algebra shows that $G_{n}(f(i))=0$ if $i \bmod 2^{n} \in\left\{1,2, \ldots, 2^{n-1}\right\}$ and $G_{n}(f(i))=1$ otherwise. Therefore, we conclude from (3) and (8) that the operator $\mathcal{B}_{n}$ defined by (6) has the form

$$
\mathcal{B}_{n} e_{i}=\left\{\begin{array}{l}
-\beta_{n} e_{i}+\beta_{n} e_{i+2^{n-1}} \\
\quad \text { if } i \bmod 2^{n} \in\left\{1,2, \ldots, 2^{n-1}\right\} \\
-\alpha_{n} e_{i}+\alpha_{n} e_{i-2^{n-1}} \\
\quad \text { if } i \bmod 2^{n} \in\left\{0,2^{n-1}+1, \ldots, 2^{n}-1\right\},
\end{array}\right.
$$

where $i \geq 1$. Since $\mathcal{B}_{n}\left(\xi_{i}\right)_{i \in \mathbb{N}}=\sum_{i \in \mathbb{N}} \xi_{i} \mathcal{B}_{n} e_{i}$, (9) implies directly (7).

For instance, $\mathcal{B}_{1}$ has the form

$$
\mathcal{B}_{1}\left(\xi_{i}\right)_{i \in \mathbb{N}}=\left(-\beta_{1} \xi_{1}+\alpha_{1} \xi_{2}, \beta_{1} \xi_{1}-\alpha_{1} \xi_{2},-\beta_{1} \xi_{3}+\alpha_{1} \xi_{4}, \beta_{1} \xi_{3}-\alpha_{1} \xi_{4}, \ldots\right)
$$

Denote

$$
\mathcal{A}_{n}=\sum_{k=1}^{n} \mathcal{B}_{k}, \quad n \geq 1 .
$$

The following corollary is a direct conclusion from Lemma 1.

Corollary 1 If $\left(\eta_{i}\right)_{i \in \mathbb{N}}=\mathcal{A}_{n}\left(\xi_{i}\right)_{i \in \mathbb{N}}$, then $\eta_{i}=\sum_{k=1}^{n} \zeta_{k}$, where $\zeta_{k}, k=1, \ldots, n$, are given by

$$
\zeta_{k}=\left\{\begin{array}{l}
-\beta_{k} \xi_{i}+\alpha_{k} \xi_{i+2^{k-1}} \\
\quad \text { if } i \bmod 2^{k} \in\left\{1,2, \ldots, 2^{k-1}\right\} \\
-\alpha_{k} \xi_{i}+\beta_{k} \xi_{i-2^{k-1}} \\
\quad \text { if } i \bmod 2^{k} \in\left\{0,2^{k-1}+1, \ldots, 2^{k}-1\right\} .
\end{array}\right.
$$

It turns out that $\mathcal{B}_{n}$ 's and $\mathcal{A}_{n}$ 's have the following interesting property.

Corollary 2 Suppose that $x \in l^{1}(\mathbb{N})$ and denote $\left(\eta_{i}\right)_{i \in \mathbb{N}}=\mathcal{B}_{n} x,\left(\eta_{i}^{\prime}\right)_{i \in \mathbb{N}}=\mathcal{A}_{n} x$. Then

$$
\sum_{i=1}^{\infty} \eta_{i}=\sum_{i=1}^{\infty} \eta_{i}^{\prime}=0
$$


Proof Due to (10), it is enough to prove $\sum_{i=1}^{\infty} \eta_{i}=0$. Since $\mathcal{B}_{n}$ is bounded, i.e. $\left\|\mathcal{B}_{n} x\right\| \leq \max \left\{\alpha_{n}, \beta_{n}\right\}\|x\|$, we have

$$
\sum_{i=1}^{\infty} \eta_{i}=\lim _{m \rightarrow \infty} \sum_{i=1}^{m} \eta_{i}=\lim _{m \rightarrow \infty}\left(\sum_{i=1}^{k 2^{n}} \eta_{i}+\sum_{i=k 2^{n}+1}^{m} \eta_{i}\right)
$$

for any $k \in \mathbb{N}$. The first sum in the above limit is 0 by Lemma 1 . Furthermore, for any $\varepsilon>0$ there exists $k_{0}$ such that $\sum_{i=k_{0}}^{\infty}\left|\eta_{i}\right|<\varepsilon$. In consequence, $\left|\sum_{i=k_{0}}^{\infty} \eta_{i}\right|<M \varepsilon$ for a certain constant $M>0$ and $\varepsilon>0$. Since $\varepsilon$ is arbitrary (11) is proved.

\section{The Operator}

We define $\mathcal{A}$ as

$$
\mathcal{A} x:=\sum_{k=1}^{+\infty} \mathcal{B}_{k} x=\lim _{n \rightarrow+\infty} \mathcal{A}_{n} x
$$

with domain

$$
\mathcal{D}(\mathcal{A})=\left\{x \in l^{1}(\mathbb{N}): \sum_{k=1}^{+\infty}\left\|\mathcal{B}_{k} x\right\|<\infty\right\} .
$$

Let $x=\left(\xi_{i}\right)_{i \in \mathbb{N}}$ be an element of $\mathcal{D}(\mathcal{A})$ and denote $\left(\eta_{i}\right)_{i \in \mathbb{N}}=\mathcal{A} x$. Since the norm convergence in $l^{1}(\mathbb{N})$ implies the coordinate-wise convergence, we conclude from Corollary 1 that

$$
\eta_{1}=\lim _{n \rightarrow+\infty} \sum_{k=1}^{n}\left(-\beta_{k} \xi_{1}+\alpha_{k} \xi_{1+2^{k-1}}\right),
$$

and if $i=2^{l}+m$ for some $l \geq 0$ and $m \in\left\{1,2, \ldots, 2^{l}\right\}$, we have

$$
\eta_{i}=\sum_{k=1}^{l+1} \zeta_{k}+\lim _{n \rightarrow+\infty} \sum_{k=l+2}^{n}\left(-\beta_{k} \xi_{i}+\alpha_{k} \xi_{i+2^{k-1}}\right)
$$

where $\zeta_{k}, k=1, \ldots, l+1$ are given by

$$
\zeta_{k}=\left\{\begin{array}{l}
-\beta_{k} \xi_{i}+\alpha_{k} \xi_{i+2^{k-1}}, \\
\quad \text { if } i \bmod 2^{k} \in\left\{1,2, \ldots, 2^{k-1}\right\}, \\
-\alpha_{k} \xi_{i}+\beta_{k} \xi_{i-2^{k-1}}, \\
\quad \text { if } i \bmod 2^{k} \in\left\{0,2^{k-1}+1, \ldots, 2^{k}-1\right\} .
\end{array}\right.
$$

Notice that a necessary condition for an $x$ to be in $\mathcal{D}(\mathcal{A})$ is

$$
\lim _{n \rightarrow+\infty}\left(-\beta_{n} \xi_{i}+\alpha_{n} \xi_{i+2^{n-1}}\right)=0
$$

for every $i \geq 1$. 
For now, one can merely infer that $0 \in \mathcal{D}(\mathcal{A})$. However, in the proof of Theorem 1 we will produce some non-trivial elements of $\mathcal{D}(\mathcal{A})$. The following theorem is the main result of this article.

Theorem 1 For the operator $\mathcal{A}$ defined by (12)-(13), the conditions in (1) are satisfied.

Proof First we show (ii). Fix $i \in \mathbb{N}$ and notice that by (2) we have

$$
\lim _{n \rightarrow+\infty} \sum_{k=l+2}^{n}\left(-\beta_{k} \xi_{i}+\alpha_{k} \xi_{i+2^{k-1}}\right)=-\lim _{n \rightarrow+\infty} \sum_{k=l+2}^{n} \beta_{k}=-\infty
$$

proving that $e_{i} \notin \mathcal{D}(\mathcal{A})$.

Next we prove (i). Let $x=\left(\xi_{1}, \xi_{2}, \ldots\right)$ be defined as follows. First we put

$$
\xi_{1}=1, \quad \xi_{1+2^{k-1}}=\frac{\beta_{k}}{\alpha_{k}}, \quad k \geq 1,
$$

and if $i=2^{l}+m$ for some $l \geq 0$ and $m \in\left\{1,2, \ldots, 2^{l}\right\}$, we define recursively

$$
\xi_{i+2^{k-1}}=\frac{\beta_{k}}{\alpha_{k}} \xi_{i}, \quad k \geq l+2 .
$$

From this, we infer that for $i=1+2^{l_{1}-1}+\ldots+2^{l_{m}-1}$ with $l_{1}<l_{2}<\ldots<l_{m}$ one can write

$$
\xi_{i+2^{k-1}}=\frac{\beta_{k}}{\alpha_{k}} \cdot \frac{\beta_{l_{m}} \beta_{l_{m}-1} \ldots \beta_{l_{1}}}{\alpha_{l_{m}} \alpha_{l_{m}-1} \ldots \alpha_{l_{1}}}, \quad k \geq l_{m}+1
$$

Then, the norm of $x$ can be expressed as

$$
\|x\|=1+\sum_{l=1}^{\infty} \frac{\beta_{l}}{\alpha_{l}}+\sum_{l_{1}<l_{2}}^{\infty} \frac{\beta_{l_{1}} \beta_{l_{2}}}{\alpha_{l_{1}} \alpha_{l_{2}}}+\sum_{l_{1}<l_{2}<l_{3}}^{\infty} \frac{\beta_{l_{1}} \beta_{l_{2}} \beta_{l_{3}}}{\alpha_{l_{1}} \alpha_{l_{2}} \alpha_{l_{3}}}+\ldots
$$

From the Problem 3.8.26 in [4] and the fact that $\sum_{l=1}^{\infty} \frac{\beta_{l}}{\alpha_{l}}<\infty$ (which follows from (2)), we have

$$
\|x\|=\prod_{l=1}^{\infty}\left(1+\frac{\beta_{l}}{\alpha_{l}}\right)<\infty
$$

meaning that $x \in l^{1}(\mathbb{N})$. To conclude that $x \in \mathcal{D}(\mathcal{A})$ we need to show that condition (13) is satisfied. To this end, denote $\left(\eta_{i}\right)_{i \in \mathbb{N}}=\mathcal{B}_{k} x$ and notice that (7) implies $\eta_{i}=$ $-\beta_{k} \xi_{i}+\alpha_{k} \frac{\beta_{k}}{\alpha_{k}} \xi_{i}=0$ or $\eta_{i}=-\alpha_{k} \xi_{i}+\beta_{k} \frac{\alpha_{k}}{\beta_{k}} \xi_{i}=0$. In other words,

$$
\sum_{k=1}^{+\infty}\left\|\mathcal{B}_{k} x\right\|=0
$$


The above means that $x \in \mathcal{D}(\mathcal{A})$ and $\mathcal{A} x=0$.

Now we construct a sequence of elements of $\mathcal{D}(\mathcal{A})$ converging to $e_{1}$. Fix $p \geq 2$ and define $y_{p}=\left(\xi_{1}, \xi_{2}, \ldots\right)$ as follows

$$
\xi_{1}=1, \quad \xi_{1+2^{k-1}}= \begin{cases}0 & , \quad k=1,2, \ldots, p-1, \\ \frac{\beta_{k}}{\alpha_{k}} & , \quad k \geq p\end{cases}
$$

and if $i=2^{l}+m$ for some $l \geq 0$ and $m \in\left\{1,2, \ldots, 2^{l}\right\}$, we define $\xi_{i+2^{k-1}}$ recursively as in (17). Observe that $\left\|y_{p}\right\| \leq\|x\|$ for every $p \geq 2$. Additionally, from (19) we have $\xi_{i}=0$ for $i=2,3, \ldots, 1+2^{p-1}$. It is clear that the coordinate-wise limit of $y_{p}$ is $e_{1}$.

Now choose any $\varepsilon>0$ and fix $M \in(0,1)$. We conclude from (2) that there exists $l_{0}$ such that $\sum_{l=l_{0}}^{\infty} \frac{\beta_{l}}{\alpha_{l}}<\varepsilon$ and $\frac{\beta_{l}}{\alpha_{l}}<M$, for all $l \geq l_{0}$. This, together with (18), implies

$$
\left\|y_{p}-e_{1}\right\| \leq \varepsilon \sum_{k=0}^{\infty} M^{k}=\frac{\varepsilon}{1-M}, \quad \text { for } p \geq l_{0} .
$$

Since $\varepsilon$ is arbitrary, $\lim _{p \rightarrow+\infty}\left\|y_{p}-e_{1}\right\|=0$.

What remains to show is $\mathcal{A} y_{p} \in l^{1}(\mathbb{N})$. From Lemma 1 , we have

$$
\mathcal{B}_{k} y_{p}=(-\beta_{k}, 0, \ldots, 0, \underbrace{\beta_{k}}_{1+2^{k-1}-t h}, 0,0, \ldots), \quad k=1,2, \ldots, p-1
$$

and $\mathcal{B}_{k} y_{p}=0$ for $k \geq p$. This implies

$$
\sum_{k=1}^{+\infty}\left\|\mathcal{B}_{k} y_{p}\right\|=2 \sum_{k=1}^{p-1} \beta_{k}<\infty, \quad p \geq 2 .
$$

So $y_{p} \in \mathcal{D}(\mathcal{A})$ for every $p \geq 2$ and

$$
\mathcal{A} y_{p}=\left(\eta_{i}\right)_{i \in \mathbb{N}}=\left\{\begin{array}{l}
-\sum_{k=1}^{p-1} \beta_{k}, \quad i=1, \\
\beta_{k}, \quad \text { for } i=1+2^{k-1} \text { and } k=1,2, \ldots, p-1, \\
0, \quad \text { otherwise. }
\end{array}\right.
$$

Incidentally, notice that $\mathcal{A} y_{p}$ has only a finite number of nonzero components and $\sum_{k=1}^{\infty} \eta_{k}=0$. To summarize, we have constructed a sequence $y_{p}, p \geq 2$, of elements of $\mathcal{D}(\mathcal{A})$ converging to $e_{1}$. 
Now we show that a similar construction can be carried out for any $e_{i}, i \geq 2$. Fix $i \geq 2$ and observe that for $x$ defined by (16)-(17) we have

$$
\left\|\frac{1}{\xi_{i}} x\right\|=\left\|(\frac{1}{\xi_{i}}, \frac{\xi_{2}}{\xi_{i}}, \ldots, \frac{\xi_{i-1}}{\xi_{i}}, \underbrace{1}_{i-t h}, \frac{\xi_{i+1}}{\xi_{i}}, \ldots)\right\|=\frac{1}{\xi_{i}}\|x\|<\infty .
$$

Suppose that $i=2^{l}+m$ for some $l \geq 0$ and $m \in\left\{1,2, \ldots, 2^{l}\right\}$. We define $z_{p}=$ $\left(\xi_{1}, \xi_{2}, \ldots\right)$ as follows. At first, let

$$
\xi_{i}=1, \quad \xi_{i+2^{k-1}}= \begin{cases}0 & , \quad k=l+2, l+3, \ldots, p-1, \\ \frac{\beta_{k}}{\alpha_{k}} \quad, \quad k \geq p\end{cases}
$$

where $p>i+2^{l+1}$. For $k=1,2, \ldots, l+1$, define

$$
\begin{cases}\xi_{i+2^{k-1}}=0, & \text { if } i \bmod 2^{k} \in\left\{1,2, \ldots, 2^{k-1}\right\}, \\ \xi_{i-2^{k-1}}=0, & \text { if } i \bmod 2^{k} \in\left\{0,2^{k-1}+1, \ldots, 2^{k}-1\right\} .\end{cases}
$$

In all other cases, elements of $z_{p}$ are defined recursively by (17).

Notice that $\xi_{j}=0$ for $j \in\left\{1,2, \ldots, i+2^{p-1}\right\} \backslash\{i\}$ and that the coordinatewise limit of $z_{p}$ is $e_{i}$. Furthermore, it is clear from (20) that $\left\|z_{p}\right\| \leq \frac{1}{\xi_{i}}\|x\|$ and that $\lim _{p \rightarrow \infty}\left\|z_{p}-e_{i}\right\|=0$. Computing $\mathcal{A} z_{p}$ in a similar way as we $\operatorname{did} \mathcal{A} y_{p}$, we see that it has only a finite number of nonzero terms, meaning that $z_{p} \in \mathcal{D}(\mathcal{A})$. This completes the proof.

\section{Conclusions}

Recall that $\left(\mathcal{B}_{n}\right)_{n \in \mathbb{N}}$ is a sequence of bounded operators defined on $l^{1}(\mathbb{N})$ and $\left(e^{t \mathcal{B}_{n}}\right)_{n \in \mathbb{N}}$ is the corresponding sequence of commuting Markov semigroups, i.e. for any $m, n \in \mathbb{N}$ and $x \in l^{1}(\mathbb{N})$, we have

$$
e^{t \mathcal{B}_{n}} e^{s \mathcal{B}_{m}} x=e^{s \mathcal{B}_{m}} e^{t \mathcal{B}_{n}} x, \quad t, s \geq 0
$$

The above follows from the fact that $\mathcal{B}_{n}$ 's are isomorphic images of $B_{n}$ 's, see (6). Hence, for every $n \in \mathbb{N}, \mathcal{T}_{n}(t)=\prod_{k=1}^{n} e^{t \mathcal{B}_{k}}$ is a strongly continuous semigroup with the generator $\mathcal{A}_{n}$ given by (10).

The following is a consequence of Theorem 1.

Corollary 3 The semigroups $\left\{\mathcal{T}_{n}(t), t \geq 0\right\}$ converge, when $n \rightarrow \infty$, to a strongly continuous Markov semigroup $\{\mathcal{T}(t), t \geq 0\}$. This limit semigroup is the Blackwell semigroup on $l^{1}(\mathbb{N})$. Furthermore, the generator $\mathcal{A}_{B}$ of $\{\mathcal{T}(t), t \geq 0\}$ is the closure of $\mathcal{A}$ given by (12)-(13). In other words, $\overline{\mathcal{A}}=\mathcal{A}_{B}$. 
Proof Theorem 2.1 of [2] specifies conditions under which an infinite product of commuting contraction semigroups exists. All those conditions are satisfied in our case (thanks to Theorem 1), especially the key one that $\mathcal{D}(\mathcal{A})$ is dense in $l^{1}(\mathbb{N})$.

Remark 1 Theorem 1 may be also considered as an alternative proof of existence of (4) to the proof presented in [2]. Since semigroups $\left\{\mathcal{T}_{n}(t), t \geq 0\right\}$ and $\left\{T_{n}(t), t \geq 0\right\}$ are isomorphic, the strong convergence of one of them implies the strong convergence of the other and vice versa. The proof of convergence of $T_{n}(t)$ presented in [2] can be termed as "a direct proof".

Corollary 4 Suppose that $x \in \mathcal{D}(\mathcal{A})$ and denote $\left(\eta_{i}\right)_{i \in \mathbb{N}}=\mathcal{A} x$, Then

$$
\sum_{i=1}^{\infty} \eta_{i}=0 .
$$

Proof Notice that $\eta_{i}$ 's are the coordinate-wise limits of $\mathcal{A}_{n} x$, see (14) and (15). Hence, (22) is a consequence of (11).

Acknowledgements I would like to thank the reviewer for her or his comments. These comments helped me to improve this article.

Open Access This article is licensed under a Creative Commons Attribution 4.0 International License, which permits use, sharing, adaptation, distribution and reproduction in any medium or format, as long as you give appropriate credit to the original author(s) and the source, provide a link to the Creative Commons licence, and indicate if changes were made. The images or other third party material in this article are included in the article's Creative Commons licence, unless indicated otherwise in a credit line to the material. If material is not included in the article's Creative Commons licence and your intended use is not permitted by statutory regulation or exceeds the permitted use, you will need to obtain permission directly from the copyright holder. To view a copy of this licence, visit http://creativecommons.org/licenses/by/4.0/.

\section{References}

1. Blackwell, D.: Another countable Markov process with only instantaneous states. Ann. Math. Stat. 29, 313-316 (1958)

2. Bobrowski, A.: On a somewhat forgotten condition of Hasegawa and on Blackwells example. Arch. Math. 104, 237-246 (2015)

3. Feller, W., McKean, H.P.: A diffusion equivalent to a countable Markov chain. Proc. Nat. Acad. Sci. USA 42, 351-354 (1956)

4. Nowak, M., Kaczor, W.: Problems in Mathematical Analysis: Real Numbers, Sequences and Series, vol. I. AMS, Providence (2000)

5. Reuter, G.E.H.: Denumerable Markov processes and the associated contraction semigroups on $l$. Acta Math. 97, 1-46 (1957)

Publisher's Note Springer Nature remains neutral with regard to jurisdictional claims in published maps and institutional affiliations. 